\title{
Discrete Decision Problems, Multiple Criteria Optimization Classes and Lexicographic Max-Ordering
}

\author{
Matthias Ehrgott ${ }^{1}$ \\ 1 Department of Mathematics, University of Kaiserslautern, PO Box 3049, 67653 Kais- \\ erslautern, Germany, e-mail: ehrgott@mathematik.uni-kl.de; partially supported by the \\ Deutsche Forschungsgemeinschaft (DFG) and grant ERBCHRXCT930087 of the European \\ HC\&M Programme
}

\begin{abstract}
The topic of this paper are discrete decision problems with multiple criteria. We first define discrete multiple criteria decision problems and introduce a classification scheme for multiple criteria optimization problems. To do so we use multiple criteria optimization classes. The main result is a characterization of the class of lexicographic max-ordering problems by two very useful properties, reduction and regularity. Subsequently we discuss the assumptions under which the application of this specific MCO class is justified. Finally we provide (simple) solution methods to find optimal decisions in the case of discrete multiple criteria optimization problems.
\end{abstract}

Keywords. Discrete decision problems, Classification, Lexicographic maxordering

\section{Discrete Decision Problems and Multiple Criteria Optimization Classes}

A discrete decision problem consists of selecting from a finite set of alternatives $A=\left\{a_{1}, \ldots, a_{m}\right\}$ an 'optimal' one. In this paper we consider the context of multiple criteria decision making (MCDM). The solution of discrete decision problems with multiple criteria is often called multiple attribute decision making, see [HY81]. Every alternative is evaluated with respect to a certain number of criteria, or objective functions. We assume that $f_{q}: A \rightarrow \mathbb{R} ; q=1, \ldots, Q$ are $Q$ real valued functions representing the different criteria. Therefore we can calculate the value of each alternative with respect to each criterion as given by $v_{q j}=f_{q}\left(a_{j}\right)$. Since the number of alternatives as well as the number of criteria is finite it is convenient to write all the values as a $Q \times m$ matrix:

$$
V=\left(v_{q j}\right)=\left(f_{q}\left(a_{j}\right)\right)
$$


Matrix $V$ summarizes the performance of all alternatives with respect to all criteria. We understand an optimal alternative to be one with minimal performance with respect to the $Q$ criteria. This implies that minima of a set of vectors, namely of $\left\{\left(f_{1}\left(a_{j}\right), \ldots, f_{Q}\left(a_{j}\right)\right): j=1, \ldots, m\right\}$, or, in other words, of the columns of $V$ have to be found. Due to the fact that, if $Q \geq 2$, there does not exist a canonical total order of $\mathbb{R}^{Q}$, there are lots of reasonable choices of a (partial) order of $\mathbb{R}^{Q}$ which will lead to as many different definitions of minima. This means that, besides specification of the criteria, the decision maker (DM) faces the task of selecting an order, which will define the optimal alternatives of the decision problem and finally he also has to solve his decision problem. It is a straightforward assumption that the DM will have certain ideas about properties of this solution process.

In the remaining part of this section we will introduce a framework which allows to formalize the notion of the DM's ideas concerning desirable features of the solution process and properties of optimal solutions of decision problems. In Section 2 we will then introduce two such desirable properties and show that they uniquely define a specific pattern of solving decision problems. The corresponding (simple) solution method will be presented in Section 3 .

Formally, let us consider a general multiple criteria optimization problem (MCOP). Let $\mathcal{F}$ be the set of feasible solutions and $f: \mathcal{F} \rightarrow \mathbb{R}^{Q}$ be the objective function. Function $f$ is a vector valued function and can be written as $f=\left(f_{1}, \ldots, f_{Q}\right)$, i.e. $f_{1}, \ldots, f_{Q}$ are the $Q$ criteria. The goal of the solution of MCOP is to find 'minimizers' of the objective function, considered to be optimal solutions of the MCOP at hand. It is quite common in MCDM that an optimal decision is found by optimizing not directly with respect to the criteria. Often a utility function $U$ is applied to the vectors $f(x), x \in \mathcal{F}$. Then any $x \in \mathcal{F}$ minimizing $U(f(x))$ is an optimal solution of the MCOP. For instance, $U$ can be a scalarizing function defined by a weighting vector $\lambda=\left(\lambda_{1}, \ldots, \lambda_{Q}\right)$ where $\lambda_{q} \geq 0$ and $\sum_{q=1}^{Q} \lambda_{Q}=1$. In this case $U$ is defined by $U(f(x))=\sum_{q=1}^{Q} \lambda_{q} f_{q}(x)$.

From these considerations we conclude that, in general, the solution of an MCOP cannot be determined from the feasible set $\mathcal{F}$ and the objective function $f$, mapping $\mathcal{F}$ to $\mathbb{R}^{Q}$, only. Information concerning the utility function $U$ and the final comparison of the feasible solutions in the image space of $U$ ( $\mathbb{R}$ with the canonical order in in the case of a scalarizing function) is needed. However, this information is usually implicitly defined by the 'ideas' of the DM about the solution of 'his' MCOP. Making this information available the DM could possibly gain a more profound understanding of the structure of an MCOP. Furthermore, it would be easier for him to communicate with others or even help him to find existing solution procedures.

We therefore propose to include this 'hidden' information explicitly in the formulation of an MCOP. Our concept is more general and comprises utility functions, scalarizing functions and many other as special cases. This concept provides the possibility of a classification of multiple criteria optimization 
problems, see [Ehr97], and also is a helpful tool for theoretical achievements in the area.

Let $\theta: \mathbb{R}^{Q} \rightarrow \mathbb{R}^{P}$ be a point-to-set map, i.e. $\theta(v) \subset \mathbb{R}^{P}$ is a subset of points in $\mathbb{R}^{P}$, where $P \geq 1$. This point-to-set map is applied to the objective values vectors $f(x)$, yielding a set of outcomes

$$
O=\bigcup_{x \in \mathcal{F}} \theta(f(x))
$$

Let us assume that $\mathbb{R}^{P}$ is endowed with some kind of ordering $\preceq$, which we assume to be at least a strict partial order. Then we define the set of minima to be all $z \in O$ such that there does not exist another point $z^{\prime} \in O$ satisfying $z^{\prime} \preceq z$ and $z^{\prime} \neq z$. An optimal solution of an MCOP is then any feasible solution $x$ such that $x$ is in the preimage of an element of the set of minima. The map $\theta$ will be called the model of the MCOP problem.

We illustrate the concept by showing that scalarizing functions mentioned above are special cases of models. If we define $\theta$ by $\theta(f(x))=\sum_{q=1}^{Q} \lambda_{q} f_{q}(x)$ and let $P=1$ we have $O=\left\{\sum_{q=1}^{Q} \lambda_{q} f_{q}(x): x \in \mathcal{F}\right\}$ and the set of minima is simply the minimum of the scalarizing function and any minimizer of that same function is considered an optimal solution, as usual. Note that $\theta$ maps to $\mathbb{R}$ and solutions are compared by comparing $\theta(f(x))$ values in the ordered set $(\mathbb{R}, \leq)$. But, moreover, our concept of the model map allows the following definition: let

$$
\Lambda:=\left\{\lambda \in \mathbb{R}^{Q}: \lambda_{q} \geq 0, q=1, \ldots, Q ; \sum_{q=1}^{Q} \lambda_{q}=1\right\} .
$$

We define

$$
\theta(f(x)):=\langle\Lambda, f(x)\rangle:=\{\langle\lambda, f(x)\rangle: \lambda \in \Lambda\} .
$$

(Here $\langle\lambda, f(x)\rangle$ denotes the Euclidean scalar product of $\lambda$ and $f(x)$ in $\mathbb{R}^{Q}$ ). By this definition the set of minima is the set of all minima with respect to any scalarizing function.

We introduce some notation and formalize the ideas of the above discussion. As mentioned earlier the basic information about the MCOP problem is given by the set of feasible solutions $\mathcal{F}$, the criteria, $f=\left(f_{1}, \ldots, f_{Q}\right)$, and the image space $\mathbb{R}^{Q}$ of $f$. We will call this triple $\left(\mathcal{F}, f, \mathbb{R}^{Q}\right)$ the data of the MCOP. The description of the MCOP is completed by the model map $\theta$ (which is possibly a point-to-set map) and the ordered set $\left(\mathbb{R}^{P}, \preceq\right)$, in which minima are actually sought. Therefore we have three basic elements defining an MCO problem, namely data, model, and ordered set. From now on MCOPs will be denoted by

$$
\left(\mathcal{F}, f, \mathbb{R}^{Q}\right) / \theta /\left(\mathbb{R}^{P}, \preceq\right) .
$$

As an indication that $P>1$ makes perfect sense we note that if we want to solve an MCOP problem in the commonly used sense of Pareto optimality (i.e. 
a solution $x^{*} \in \mathcal{F}$ is Pareto optimal if there does not exist another solution $x \in \mathcal{F}$ such that $f_{q}(x) \leq f_{q}\left(x^{*}\right)$ for all $q=1, \ldots, Q$ and $f_{j}(x)<f_{j}\left(x^{*}\right)$ for at least one $j$ ). We choose $\theta=$ id, the identity map, $\mathbb{R}^{P}=\mathbb{R}^{Q}$ and $<$ for $\preceq$. Here we use $<$ in the following way: if $a, b \in \mathbb{R}^{Q}$ then $a<b$ if $a_{q} \leq b_{q}$ for all $q=1, \ldots, Q$ and $a_{j}<b_{j}$ for at least one $j$. Thus $\left(\mathcal{F}, f, \mathbb{R}^{Q}\right) / \mathrm{id} /\left(\mathbb{R}^{Q},<\right)$ denotes an MCOP to be solved in the sense of Pareto optimality.

The solution of an $\operatorname{MCOP}\left(\mathcal{F}, f, \mathbb{R}^{Q}\right) / \theta /\left(\mathbb{R}^{P}, \preceq\right)$ consists of two parts: the determination of the set of minima of $\theta(f(\mathcal{F}))$ and the determination of the set of optimal solutions, i.e. the preimage of the set of minima. The former set will be denoted by

$$
\mathcal{V}_{\text {opt }}\left(\left(\mathcal{F}, f, \mathbb{R}^{Q}\right) / \theta /\left(\mathbb{R}^{P}, \preceq\right)\right)
$$

and the latter by

$$
\text { Opt }\left(\left(\mathcal{F}, f, \mathbb{R}^{Q}\right) / \theta /\left(\mathbb{R}^{P}, \preceq\right)\right) \text {. }
$$

The notation of MCOP we have introduced here can be used as a classification scheme for multiple criteria optimization problems. We refer to [Ehr97] for more about this aspect. The classification makes it easily possible to compare various MCOP problems and to exhibit their interrelations. It may also provide a structure and guiding line through the vast amount of literature on multiple criteria optimization. A second main advantage of the previously introduced notation is the possibility of proving results which hold independent of the data of a specific problem. In [Ehr97] various results of this type have been proven. They can be interpreted as structural properties of whole classes of MCOPs. We follow [Ehr97] and define an MCO class to comprise the set of all MCOPs which have the same model map and ordered set. An MCO class is denoted by

$$
(\bullet, \bullet, \bullet) / \theta /\left(\mathbb{R}^{P}, \preceq\right) .
$$

The dots indicate an arbitrary entry. We note that results which can be shown for an MCO class are valid for all problems in the class, i.e. for any specific problem data.

In Section 2 we introduce properties of MCO classes which are reasonable from a decision makers point of view. We have mentioned the 'ideas' of the DM about the solution of MCOPs earlier. Formally, these 'ideas' are properties of $\mathrm{MCO}$ classes. For two of these properties we show that they characterize a specific MCO class, the lexicographic max-ordering MCO class. Subsequently further properties of this class are established. Section 2 is concluded by a discussion of the circumstances under which the application of this specific MCO class seems to be justified. Section 3 is devoted to (simple) solution procedures for determining optimal decisions under the lex-MO MCO class when the MCOP is a discrete multiple criteria decision problem. 


\section{Reduction, Regularity and the Lexicographic Max-Ordering MCO Class}

In this section we propose the lexicographic max-ordering MCO class. This class will be shown to be uniquely defined by two properties which we describe before proving the main result. Throughout this paper we only consider MCO classes which satisfy the following normalization property. For the special case that $Q=1$ it is natural to assume that the usual single objective minimization with the canonical order of $\mathbb{R}$ should be applied. Therefore, if $(\bullet, \bullet, \bullet) / \operatorname{sort} /\left(\mathbb{R}^{P}, \preceq\right)$ is any MCO class, we require that $Q=1$ implies

$$
(\bullet, \bullet, \mathbb{R}) / \operatorname{sort} /\left(\mathbb{R}^{P}, \preceq\right)=(\bullet, \bullet, \mathbb{R}) / \mathrm{id} /(\mathbb{R}, \leq),
$$

which actually means ordinary minimization of one criterion. Note that the MCO classes defined by Pareto optimality $(\bullet, \bullet, \bullet) /$ id $/\left(\mathbb{R}^{Q},<\right)$ and by scalarizing the criteria $(\bullet, \bullet, \bullet) /\langle\lambda,\rangle /.(\mathbb{R}, \leq)$ both satisfy the normalization property. For other MCO classes we refer to [Ehr97].

Let $(\bullet, \bullet, \bullet) / \theta /\left(\mathbb{R}^{P}, \preceq\right)$ be any MCO class. What could be desirable properties from a decision makers point of view? We propose two. The first one is related to a reduced optimization problem. Let us suppose that, for whatever reason, for some objective functions the values that are taken for some solutions in Opt $\left((\bullet, \bullet, \bullet) / \theta /\left(\mathbb{R}^{P}, \preceq\right)\right)$ are known. Then it should suffice to consider for the minimization only the remaining objectives, with the additional constraints that for the known objectives the known values are taken. If the optimal solutions of this reduced minimization problem are exactly those of the original problem which have the given values for the specified objectives, we say that the MCO class satisfies the reduction property. Formally we define the reduced problem as follows.

Definition 1 Let $(\bullet, \bullet, \bullet) / \theta /\left(\mathbb{R}^{P}, \preceq\right)$ be a MCO class and let $\left(\mathcal{F}, f, \mathbb{R}^{Q}\right)$ be data to define any $M C O$ problem of the given MCO class. Let $y \in \mathbb{R}^{Q}$ be such that there exists at least one $x \in \operatorname{Opt}\left(\left(\mathcal{F}, f, \mathbb{R}^{Q}\right) / \theta /\left(\mathbb{R}^{P}, \preceq\right)\right)$ with $f(x)=y$. Furthermore let $\mathcal{K}:=\left\{i_{1}, \ldots, i_{k}\right\} \subseteq \mathcal{Q}$ be an index set. Then the reduced problem for $\mathcal{K}$, denoted by $R P(\mathcal{K})$, where $f^{\mathcal{K}}:=\left(f_{i_{1}}, \ldots, f_{i_{k}}\right)$ is defined by

$$
\left(\mathcal{F}^{\mathcal{K}}, f^{\mathcal{K}}, \mathbb{R}^{k}\right) / \theta /\left(\mathbb{R}^{P}, \preceq\right)
$$

and $\mathcal{F}^{\mathcal{K}}:=\left\{x \in \mathcal{F}: f_{q}(F)=y_{q} \forall q \in \mathcal{Q} \backslash \mathcal{K}\right\}$.

Thus, in the reduced problem the values of the functions with indices in $\mathcal{Q} \backslash \mathcal{K}$ are fixed, those with indices in $\mathcal{K}$ are still to be minimized. Using the definition of the reduced problem, we define the reduction property.

Definition 2 We say that an $M C O$ class $(\bullet, \bullet, \bullet) / \theta /\left(\mathbb{R}^{P}, \preceq\right)$ satisfies the reduction property if for all $M C O$ problem data $\left(\mathcal{F}, f, \mathbb{R}^{Q}\right)$ defining problems in the class the following holds: for all index sets $\mathcal{K} \subseteq \mathcal{Q}$ and for all 


$$
\begin{aligned}
&\left(y_{1}, \ldots, y_{Q}\right) \in \mathbb{R}^{Q} \text { as in Definition } 1 \\
& \operatorname{Opt}\left(\left(\mathcal{F}^{\mathcal{K}}, f^{\mathcal{K}}, \mathbb{R}^{k}\right) / \theta /\left(\mathbb{R}^{P}, \preceq\right)\right)= \\
&\left\{x \in \operatorname{Opt}\left(\left(\mathcal{F}, f, \mathbb{R}^{Q}\right) / \theta /\left(\mathbb{R}^{P}, \preceq\right)\right): f_{q}(x)=y_{q} \forall q \notin \mathcal{K}\right\} .
\end{aligned}
$$

The second property can be interpreted as a pessimistic point of view of the DM: he will at least want to have a solution such that the worst criterion is as good as possible. I.e. an optimal solution of an MCOP should be such that

$$
\max _{q=1, \ldots, Q} f_{q}\left(x^{*}\right) \leq \max _{q=1, \ldots, Q} f_{q}(x) \forall x \in \mathcal{F} .
$$

Evidently, minimizing the maximum of the $Q$ criteria is again an MCO class. Let $\theta(f(x)):=\max _{q=1, \ldots, Q} f_{q}(x)$ and $\left(\mathbb{R}^{P}, \preceq\right):=(\mathbb{R}, \leq)$. The corresponding $\mathrm{MCO}$ class is

$$
(\bullet, \bullet, \bullet) / \max /(\mathbb{R}, \leq)
$$

and is called max-ordering MCO class. Formally, the pessimistic attitude of a DM is formulated as the regularity property.

Definition 3 An $M C O$ class $(\bullet, \bullet, \bullet) / \theta /\left(\mathbb{R}^{P}, \preceq\right)$ satisfies the regularity property if

$$
\operatorname{Opt}\left(\left(\mathcal{F}, f, \mathbb{R}^{Q}\right) / \theta /\left(\mathbb{R}^{P}, \preceq\right)\right) \subseteq \operatorname{Opt}\left(\left(\mathcal{F}, f, \mathbb{R}^{Q}\right) / \max /(\mathbb{R}, \leq)\right) .
$$

for all $M C O$ problems $\left(\mathcal{F}, f, \mathbb{R}^{Q}\right) / \theta /\left(\mathbb{R}^{P}, \preceq\right)$ in the class.

The main purpose of this section is to show that reduction and regularity property uniquely define the lexicographic max-ordering MCO class. We will now provide the definition of this class. First, we have to define the model map.

Definition 4 For any element $x \in \mathbb{R}^{Q}$ we define

$$
\operatorname{sort}(x)=\left(\operatorname{sort}_{1}(x), \ldots, \operatorname{sort}_{Q}(x)\right)
$$

to be the vector containing the components of $x$ in non-increasing order: $\operatorname{sort}_{1}(x) \geq \ldots \geq \operatorname{sort}_{Q}(x),\left\{x_{1}, \ldots, x_{Q}\right\}=\left\{\operatorname{sort}_{1}(x), \ldots, \operatorname{sort}_{Q}(x)\right\}$.

Since sort is a mapping sort $: \mathbb{R}^{Q} \rightarrow \mathbb{R}^{Q}$ it remains to specify the order. We shall use the lexicographic order. Therefore the ordered set is $\left(\mathbb{R}^{Q}, \leq_{l e x}\right)$ and the lexicographic max-ordering class is defined by

$$
(\bullet, \bullet, \bullet) / \text { sort } /\left(\mathbb{R}^{Q}, \leq_{l e x}\right) .
$$

We will usually call that class the lex-MO MCO class for short. By this definition, a feasible solution $x^{*} \in \mathcal{F}$ of an MCOP is a lex-MO solution if its objective function vector is a lexicographically minimal element of $\operatorname{sort}(f(\mathcal{F}))$, i.e. if

$$
\operatorname{sort}\left(f\left(x^{*}\right)\right) \leq_{l e x} \operatorname{sort}(f(x)) \quad \forall x \in \mathcal{F} .
$$


This definition of optimality has appeared in the literature earlier, although with different names, see e.g. [Ogr94] [MO92] and [Beh86, Beh81]. We also refer to related papers [CLT96] and [Beh77] In none of these papers the relevance for general multiple criteria optimization has been considered. This was done for the first time in [Ehr95].

To prove our main result we first show that $(\bullet, \bullet, \bullet) /$ sort $/\left(\mathbb{R}^{Q}, \leq_{l e x}\right)$ does indeed satisfy the reduction and regularity property.

Theorem 1 The lex-MO MCO class $(\bullet, \bullet, \bullet) /$ sort $/\left(\mathbb{R}^{Q}, \leq_{\text {lex }}\right)$ satisfies reduction and regularity property.

\section{Proof:}

We will write Opt and Opt $(R P(\mathcal{K}))$ for the optimal sets of original and reduced problem in the proof. First we show that $(\bullet, \bullet, \bullet) / \operatorname{sort} /\left(\mathbb{R}^{Q}, \leq_{l e x}\right)$ satisfies the regularity property. Let $\left(\mathcal{F}, f, \mathbb{R}^{Q}\right)$ be data of an MCOP of the lex-MO class and let $x^{*} \in \mathbf{O p t}\left(\left(\mathcal{F}, f, \mathbb{R}^{Q}\right) /\right.$ sort $\left./\left(\mathbb{R}^{Q}, \leq_{l e x}\right)\right)$ be an optimal solution. Assume there exists some feasible solution $x$ such that

$$
\max _{q=1, \ldots, Q} f_{q}(x)<\max _{q=1, \ldots, Q} f_{q}\left(x^{*}\right) .
$$

Then $\operatorname{sort}_{1}(f(x))<\operatorname{sort}_{1}\left(f\left(x^{*}\right)\right)$. This is a contradiction to the choice of $x^{*}$ because it obviously implies sort $(f(x))<_{\text {lex }} \operatorname{sort}\left(f\left(x^{*}\right)\right)$.

To prove that the lex-MO class satisfies the reduction property, too, consider any MCO problem $\left(\mathcal{F}, f, \mathbb{R}^{Q}\right) /$ sort $/\left(\mathbb{R}^{Q}, \leq_{\text {lex }}\right)$. We have to show that

$$
\operatorname{Opt}(R P(\mathcal{K}))=\left\{x \in \mathbf{O p t}: f_{q}(x)=y_{q} \quad \forall q \notin \mathcal{K}\right\}
$$

for every choice of $\mathcal{K}$ and $\left(y_{1}, \ldots, y_{Q}\right)$ as in Definition 1 . We denote the latter set in (1) by $\mathbf{O p t}^{*}$ for brevity. We observe that for all solutions $x \in \mathbf{O p t}^{*}$ it holds that

$$
f_{q}(x)=f_{q}(\bar{x})=y_{q} \quad \forall q \in\{1, \ldots, Q\} \backslash \mathcal{K} \quad \forall \bar{x} \in \operatorname{Opt}(R P(\mathcal{K}))
$$

by the definition of the reduced problem.

To show (1) first let $\bar{x}$ be an element of $\operatorname{Opt}(R P(\mathcal{K}))$. By definition of Opt* it follows that

$$
\operatorname{sort}(f(x)) \leq_{l e x} \operatorname{sort}(f(\bar{x})) \quad \forall x \in \mathbf{O p t}^{*} .
$$

From (3) and (2) we conclude that

$$
\operatorname{sort}\left(f^{\mathcal{K}}(x)\right) \leq_{\text {lex }} \operatorname{sort}\left(f^{\mathcal{K}}(\bar{x})\right)
$$

which due to the choice of $\bar{x}$ as an optimal solution of the reduced problem must be satisfied with equality. Therefore we have shown that sort $(f(x))=$ sort $(f(\bar{x}))$ which clearly implies $\bar{x} \in \mathbf{O p t}^{*}$. 
We proceed with the reverse inclusion. Let $x$ be an element of Opt* . Note that $x^{*}$ is feasible for the reduced problem by definition of $R P(\mathcal{K})$. Now assume the existence of a solution $\bar{x} \in \mathbf{O p t}(R P(\mathcal{K}))$ such that

$$
\operatorname{sort}\left(f^{\mathcal{K}}(\bar{x})\right)<_{l e x} \operatorname{sort}\left(f^{\mathcal{K}}(x)\right) .
$$

This implies that also $\operatorname{sort}(f(\bar{x}))<_{l e x} \operatorname{sort}(f(x))$, contradicting the choice of $x$. Hence it must hold that

$$
\operatorname{sort}(f(\bar{x})) \leq_{l e x} \operatorname{sort}(f(x)) .
$$

(3) and (4) imply that $\operatorname{sort}(f(x))=\operatorname{sort}(f(\bar{x}))$, therefore $x$ belongs to the set Opt $(R P(\mathcal{K}))$. We have proven both inclusions in (1) and the proof is complete.

We proceed to prove the converse of Proposition 1 and thus the main result of the paper, namely that reduction and regularity are characteristic properties of the lex-MO class. Theorem 2 has first been proven in [Ehr96] and is also contained in [Ehr97].

Theorem 2 An $M C O$ class $(\bullet, \bullet, \bullet) / \theta /\left(\mathbb{R}^{P}, \preceq\right)$ satisfies reduction and regularity property if and only if

$$
(\bullet, \bullet, \bullet) / \theta /\left(\mathbb{R}^{P}, \preceq\right)=(\bullet, \bullet, \bullet) / \text { sort } /\left(\mathbb{R}^{Q}, \leq_{l e x}\right) .
$$

\section{Proof:}

Due to Theorem 1 we only have to show the 'only if' part. Let us assume that $(\bullet, \bullet, \bullet) / \theta /\left(\mathbb{R}^{P}, \preceq\right)$ is an MCO class satisfying both reduction and regularity property.

Let $\left(\mathcal{F}, f, \mathbb{R}^{Q}\right)$ be data of an MCOP of class $(\bullet, \bullet, \bullet) / \theta /\left(\mathbb{R}^{P}, \preceq\right)$. We prove the result by induction on the number $Q$ of criteria. For $Q=1$ the result follows immediately from the normalization property, since all MCO classes coincide in that case.

Let us now assume that the result holds for not more than $Q-1$ criteria and consider the case of $Q$ criteria. We have to show that

$$
\operatorname{Opt}\left(\left(\mathcal{F}, f, \mathbb{R}^{Q}\right) / \theta /\left(\mathbb{R}^{P}, \preceq\right)\right)=\operatorname{Opt}\left(\left(\mathcal{F}, f, \mathbb{R}^{Q}\right) / \operatorname{sort} /\left(\mathbb{R}^{Q}, \leq_{l e x}\right)\right) .
$$

Let $\bar{x}$ be a solution in $\operatorname{Opt}\left(\left(\mathcal{F}, f, \mathbb{R}^{Q}\right) / \theta /\left(\mathbb{R}^{p}, \preceq\right)\right)$. Furthermore, denote by $y$ the optimal value of a max-ordering solution: $y$ is the unique point in $\mathcal{V}_{\text {opt }}\left(\left(\mathcal{F}, f, \mathbb{R}^{Q}\right) / \max /(\mathbb{R}, \leq)\right)$. Since $(\bullet, \bullet, \bullet) / \theta /\left(\mathbb{R}^{P}, \preceq\right)$ satisfies the regularity property there must exist an index $q^{*}$ such that $f_{q^{*}}(\bar{x})=y$. Therefore $\bar{x} \in\left\{x \in \mathbf{O p t}\left(\left(\mathcal{F}, f, \mathbb{R}^{Q}\right) / \theta /\left(\mathbb{R}^{p}, \preceq\right)\right): f_{q^{*}}(x)=y\right\}$.

Now we consider the reduced problem with $\mathcal{K}=\{1, \ldots, Q\} \backslash\left\{q^{*}\right\}$ and the value of $f_{q^{*}}$ fixed at $y$. Then

$$
\left\{x \in \operatorname{Opt}\left(\left(\mathcal{F}, f, \mathbb{R}^{Q}\right) / \theta /\left(\mathbb{R}^{P}, \preceq\right)\right): f_{q^{*}}(x)=y\right\}
$$




$$
\begin{aligned}
& =\operatorname{Opt}\left(\left(\mathcal{F}^{\mathcal{K}}, f^{\mathcal{K}}, \mathbb{R}^{Q-1}\right) / \theta /\left(\mathbb{R}^{P}, \preceq\right)\right) \\
& =\operatorname{Opt}\left(\left(\mathcal{F}^{\mathcal{K}}, f^{\mathcal{K}}, \mathbb{R}^{Q-1}\right) / \text { sort } /\left(\mathbb{R}^{Q-1}, \leq_{l e x}\right)\right) \\
& =\left\{x \in \operatorname{Opt}\left(\left(\mathcal{F}, f, \mathbb{R}^{Q}\right) / \text { sort } /\left(\mathbb{R}^{Q}, \leq_{l e x}\right)\right): f_{q^{*}}=y\right\} \\
& \subseteq \operatorname{Opt}\left(\left(\mathcal{F}, f, \mathbb{R}^{Q}\right) / \text { sort } /\left(\mathbb{R}^{Q}, \leq_{l e x}\right)\right) .
\end{aligned}
$$

The first equation follows from the reduction property for $(\bullet, \bullet, \bullet) / \theta /\left(\mathbb{R}^{P}, \preceq\right)$. The second follows from the induction hypothesis since $R P(\mathcal{K})$ is a multiple criteria optimization problem with $Q-1$ objective functions and feasible set $\mathcal{F}^{\mathcal{K}}$ as defined in Definition 1. Finally the third is implied by the reduction property for $(\bullet, \bullet, \bullet) /$ sort $/\left(\mathbb{R}^{Q}, \leq_{l e x}\right)$. The inclusion is trivial. Hence we have shown $\bar{x} \in \mathbf{O p t}\left(\left(\mathcal{F}, f, \mathbb{R}^{Q}\right) /\right.$ sort $\left./\left(\mathbb{R}^{Q}, \leq_{l e x}\right)\right)$.

Proving the converse inclusion analogously completes the proof of $(5)$. We let $\bar{x}$ be an element of $\operatorname{Opt}\left(\left(F, f, \mathbb{R}^{Q}\right) /\right.$ sort $\left./\left(\mathbb{R}^{Q}, \leq_{l e x}\right)\right)$. By regularity we see that there exist $q^{*}$ and $y$ such that

$$
\bar{x} \in\left\{x \in \mathbf{O p t}\left(\left(F, f, \mathbb{R}^{Q}\right) / \text { sort } /\left(\mathbb{R}^{Q}, \leq_{l e x}\right)\right): f_{q^{*}}(x)=y\right\} .
$$

As above we conclude that $\bar{x} \in \mathbf{O p t}\left(\left(\mathcal{F}, f, \mathbb{R}^{Q}\right) / \theta /\left(\mathbb{R}^{P}, \preceq\right)\right)$.

The interpretation of Theorem 2 is that, if the DM agrees with the reduction property and has a pessimistic point of view (regularity property), he must choose sort as model map and the lexicographic order of $\mathbb{R}^{Q}$. In other words, he will have to solve problems of the lexicographic max-ordering MCO class. In Theorem 3 we summarize further properties of the lex-MO class.

Theorem 3 Let $\left(\mathcal{F}, f, \mathbb{R}^{Q}\right) /$ sort $/\left(\mathbb{R}^{Q}, \leq_{\text {lex }}\right)$ be a problem of the lex-MO class. Then the following hold.

(i) The set of minima $\mathcal{V}_{\text {opt }}\left(\left(\mathcal{F}, f, \mathbb{R}^{Q}\right) /\right.$ sort $\left./\left(\mathbb{R}^{Q}, \leq_{l e x}\right)\right)$ is a singleton.

(ii) $\operatorname{Opt}\left(\left(\mathcal{F}, f, \mathbb{R}^{Q}\right) / \operatorname{sort} /\left(\mathbb{R}^{Q}, \leq_{\text {lex }}\right)\right) \subseteq \operatorname{Opt}\left(\left(\mathcal{F}, f, \mathbb{R}^{Q}\right) / \mathrm{id} /\left(\mathbb{R}^{Q},<\right)\right)$.

(iii) Let $\pi$ be a permutation of $\{1, \ldots, Q\}$. Define the corresponding permutation of objective functions $f_{\pi}(x):=\left(f_{\pi(1)}(x), \ldots, f_{\pi(Q)}(x)\right)$. Then

$$
\begin{gathered}
\operatorname{Opt}\left(\left(\mathcal{F}, f, \mathbb{R}^{Q}\right) / \text { sort } /\left(\mathbb{R}^{Q}, \leq_{l e x}\right)\right)= \\
\operatorname{Opt}\left(\left(\mathcal{F}, f_{\pi}, \mathbb{R}^{Q}\right) / \text { sort } /\left(\mathbb{R}^{Q}, \leq_{l e x}\right)\right) .
\end{gathered}
$$

(iv) Let $\tau: \mathbb{R} \rightarrow \mathbb{R}$ be a strictly monotone mapping. Define the function $f_{\tau q}:=\tau \circ f_{q} \quad q=1, \ldots, Q$ and define $f_{\tau}:=\left(f_{\tau 1}, \ldots, f_{\tau Q}\right)$. Then

$$
\begin{aligned}
& \operatorname{Opt}\left((\bullet, f, \bullet) / \text { sort } /\left(\mathbb{R}^{Q}, \leq_{l e x}\right)\right)= \\
& \quad \operatorname{Opt}\left(\left(\bullet, f_{\tau}, \bullet\right) / \operatorname{sort} /\left(\mathbb{R}^{Q}, \leq_{l e x}\right)\right) .
\end{aligned}
$$

\section{Proof:}

Let $\left(\mathcal{F}, f, \mathbb{R}^{Q}\right) /$ sort $/\left(\mathbb{R}^{Q}, \leq_{l e x}\right)$ be a MCO problem of the lex-MO class. 
(i) The first assertion is clear from the definition of the lex-MO class, because the lexicographic order is a total order.

(ii) Let $x^{*} \in \operatorname{Opt}\left(\left(\mathcal{F}, f, \mathbb{R}^{Q}\right) /\right.$ sort $\left./\left(\mathbb{R}^{Q}, \leq_{l e x}\right)\right)$ be a lex-MO solution. Assume there exists some feasible solution $x$ such that $f(x)<f\left(x^{*}\right)$. It follows that $\operatorname{sort}(f(x)) \leq_{l e x} \operatorname{sort}\left(f\left(x^{*}\right)\right)$, with $\operatorname{sort}(f(x)) \neq \operatorname{sort}\left(f\left(x^{*}\right)\right)$, contradicting the choice of $x^{*}$.

(iii) Clearly $\operatorname{sort}(f(x))=\operatorname{sort}\left(f_{\pi}(x)\right)$ holds for all feasible solutions $x \in \mathcal{F}$.

(iv) By the strict monotonicity of $\tau$ it holds that $f_{q}(x)<f_{r}(x)$ if and only if $\tau\left(f_{q}(x)\right) \leq \tau\left(f_{r}(x)\right)$ for all $x \in \mathcal{F}$.

Besides the results of Theorem 3 it can also be shown that lex-MO solutions can be used to parametrize Pareto optimal solutions. We refer to [Ehr95] for details. We will now discuss the relevance of Theorem 3 for decision makers. First we note that (ii) means that lex-MO solutions are always Pareto optimal. Pareto optimality is quite natural a requirement of optimal solutions of MCOPs. If a solution which is not Pareto optimal represents an optimal decision it would be possible to find another one which is not worse with respect to all criteria an d strictly better with respect to at least one. Such a situation would raise the suspicion that the MCOP is not formulated correctly. Beyond this positive aspect, the fact that the set of minima is a singleton (i) provides an improvement to Pareto optimality: the value of a lex-MO solution is uniquely defined. The set of Pareto optimal solutions is often prohibitively large to define an optimal solution, it can even be shown that every feasible solution is Pareto optimal in some cases.

Results (iii) and (iv) will help us to identify situations in which the application of lex-MO MCO class problems is appropriate. Note that due to (iii) the numbering of criteria is irrelevant for the optimal solution. Therefore we must assume the DM's indifference with respect to the criteria. Indifference implies that there are no rankings of criteria or relative importances present, not even implicitly. In these cases we would consider lexicographic or scalarized optimization the better choice. See also the definition of $\lambda$-extremal and (global) lexicographic MCO classes in [Ehr97]. Finally, invariance under monotone transformations, implies that performance with respect to the criteria can be measured on ordinal scales. As a conclusion we state that the lex-MO MCO class is the appropriate tool when the DM is totally indifferent with respect to the criteria and performance is measured (at least) on ordinal scales. Both are, as we conjecture, rather weak assumptions.

Moreover, the sorting of the criteria suggests that the scales should be the same for all criteria. If this is not the case a priori a normalization can be applied. For instance, we can define

$$
v_{q j}^{\prime}:=\frac{\max _{j=1, \ldots, m} v_{q j}-v_{q j}}{\max _{j=1, \ldots, m} v_{q j}-\min _{j=1, \ldots, m} v_{q j}} .
$$


Then all values $v_{q j}^{\prime}$ are in the interval $[0,1]$. We refer to [HY81] for more on normalization of scales.

\section{Optimality for Discrete Decision Problems}

In this paper we will focus on discrete problems, as we have already indicated in Section 1. In this case note that the set of values of the criteria is finite. We let $R=\left\{v_{q j}: j=1, \ldots, m ; q=1, \ldots, Q\right\}$. Then for all $a_{j} \in A$ we have that $v\left(a_{j}\right) \in R^{Q}$. Therefore we will replace $\mathbb{R}^{Q}$ by the finite set $R^{Q}$. Also, since all value vectors are explicitly given via the performance matrix $V$, there is no need to consider the criteria as objective functions (this is of course needed in general multiple objective optimization). It is therefore possible to combine $f$ and $R^{Q}$ and only note $V$ instead, in the above notation for MCOPs. We will use the notation $v\left(a_{j}\right)$ or $v_{j}$ for the columns of $V$. Thus, a multiple criteria discrete decision problem is denoted by

$$
(A, V) / \theta /\left(\mathbb{R}^{P}, \preceq\right) .
$$

The following assumption guarantees that the problem is not trivial. Define

$$
v_{q}^{*}:=\min _{j=1, \ldots, Q} v_{q j} .
$$

Then the vector $v^{*}=\left(v_{1}^{*}, \ldots, v_{Q}^{*}\right)$ is called the utopia point and, clearly, if for some alternative $a_{j}$ it holds that $v\left(a_{j}\right)=v^{*}$ only this point, the ideal solution, can be considered as an optimal decision. We therefore assume that an ideal solution does not exist.

We now propose two approaches to solve a discrete multiple criteria decision problem of the lex-MO class. The first is to sort the vectors $v_{j}$ and compare them lexicographically.

\section{Algorithm 1 for $(A, V) / \operatorname{sort} /\left(\mathbb{R}^{Q}, \leq_{\text {lex }}\right)$}

Input: performance matrix $V$

Output: set of optimal decisions $O p t$

$$
\begin{aligned}
& \text { for } j=1, \ldots, m \text { do } v_{j}^{\prime}:=\operatorname{sort}\left(v_{j}\right) \\
& \min :=1 \\
& i:=1 \\
& \text { while } i \leq m \text { do } \\
& \text { begin } \\
& \text { if } v_{i}^{\prime}<_{l e x} v_{\min }^{\prime} \\
& \text { then } \min :=i \\
& i:=i+1 \\
& \text { end } \\
& O p t:=\left\{a_{j}: v_{j}^{\prime}=v_{\min }^{\prime}\right\}
\end{aligned}
$$


The time needed for this algorithm is bounded by $O(m Q \log Q)$ for the sorting of the $m$ vectors $v_{j}$. Finding the lexicographic minimum of the sorted vectors $v_{j}^{\prime}$ can be done in $O(m Q)$ time. The second algorithm uses performance matrix $V$, finds the smallest maxima of the columns and constructs a smaller matrix. This step is applied iteratively.

\section{Algorithm 2 for $(A, V) / \operatorname{sort} /\left(\mathbb{R}^{Q}, \leq_{l e x}\right)$}

Input: performance matrix $V$

Output: set of optimal decisions $O p t$

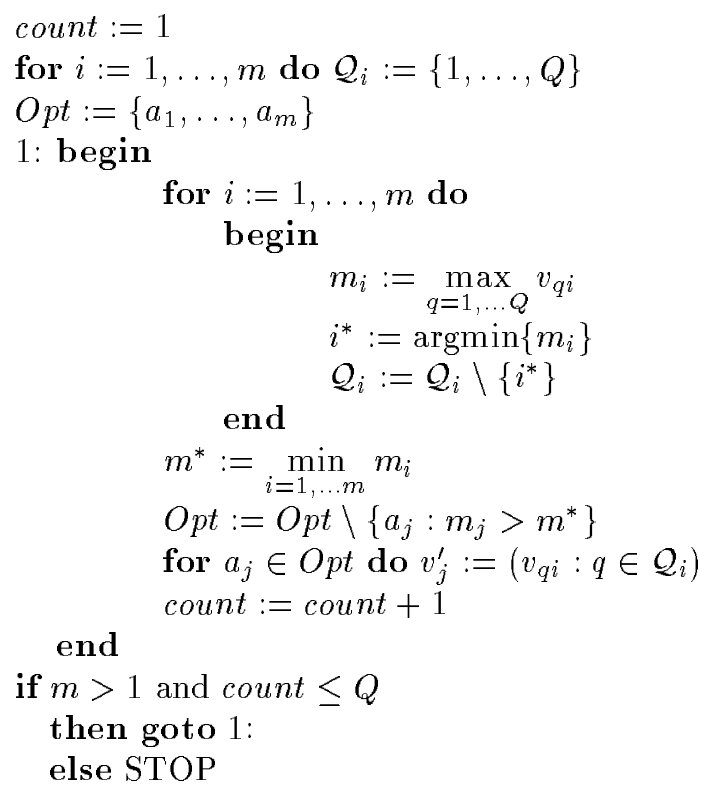

In each iteration $m$ maxima of $Q$ numbers are determined in $O(Q m)$ time. Due to the condition count $\leq Q$ and the fact that the cardinality of all $\mathcal{Q}_{i}$, for which $a_{i}$ remains in $O p t$, decreases at least by one in each iteration the algorithm stops after at most $Q$ iterations. Therefore the overall time needed is $O\left(m Q^{2}\right)$.

Comparing both algorithms, we see that in the worst case the second one is slower, however, for the first the running time is always $O(m Q \log Q)$, even in the best case. The second one on the other hand may be much faster in practice, because $O p t$ tends to become a singleton after only a few iterations.

We illustrate the methods with a small example. Let us assume somebody wants to buy a new car. There are five models $a_{1}$ to $a_{5}$ available and the relevant criteria for the decision are reputation of the brand (c1), petrol consumption (c2), price (c3), design (c4), and tenure of the guarantee (c5). To have a common ordinal scale for the criteria, she/he decides to evaluate 
the cars with respect to the criteria on a 10 point scale, the fewer points the better. Results are displayed in the matrix below

\begin{tabular}{c|ccccc} 
& $a_{1}$ & $a_{2}$ & $a_{3}$ & $a_{4}$ & $a_{5}$ \\
\hline$(\mathrm{c} 1)$ & 5 & 1 & 7 & 4 & 4 \\
$(\mathrm{c} 2)$ & 4 & 4 & 3 & 5 & 2 \\
$(\mathrm{c} 3)$ & 7 & 3 & 1 & 6 & 1 \\
$(\mathrm{c} 4)$ & 3 & 5 & 2 & 1 & 1 \\
$(\mathrm{c} 5)$ & 2 & 1 & 2 & 3 & 5
\end{tabular}

According to the first algorithm we would sort all the columns of this matrix $V$ resulting in

$$
\left(\begin{array}{lllll}
7 & 5 & 7 & 6 & 5 \\
5 & 4 & 3 & 5 & 4 \\
4 & 3 & 2 & 4 & 2 \\
3 & 1 & 2 & 3 & 1 \\
2 & 1 & 1 & 1 & 1
\end{array}\right)
$$

Finding the lexicographic minimum of the columns would yield the optimal decision $O p t=\left\{a_{5}\right\}$.

Let us now apply the second method. In the first iteration we find $m_{1}=$ $7, m_{2}=5, m_{3}=7, m_{4}=6$, and $m_{5}=5$. Therefore we would delete $a_{1}, a_{3}$, and $a_{4}$ from $O p t$. Furthermore $\mathcal{Q}_{2}=\{1,2,3,5\}$ and $\mathcal{Q}_{5}=\{1,2,3,4\}$. In the second iteration we therefore consider a much smaller problem, shown in the following matrix

$$
\left(\begin{array}{ll}
1 & 4 \\
4 & 2 \\
3 & 1 \\
1 & 1
\end{array}\right)
$$

We now find 4 as the maximal entry in both columns, hence cannot change Opt but make the problem still smaller since $\mathcal{Q}_{2}=\{1,3,5\}$ and $\mathcal{Q}_{5}=$ $\{2,3,4\}$. In the third iteration we therefore consider

$$
\left(\begin{array}{ll}
1 & 2 \\
3 & 1 \\
1 & 1
\end{array}\right)
$$

Now $m_{2}=3>m_{5}=2, a_{2}$ is deleted from $O p t$, which contains only a single element and the algorithm stops.

\section{References}

[Beh77] F.A. Behringer. On optimal decisions under complete ignorance a new criterion stronger than both Pareto and maxmin. European Journal of Operational Research, 1:295-306, 1977. 
[Beh81] F.A. Behringer. A simplex based algorithm for the lexicographically extended linear maxmin problem. European Journal of Operational Research, 7:274-283, 1981.

[Beh86] F.A. Behringer. Linear multiobjective maxmin optimization and some Pareto and lexmaxmin extensions. OR Spektrum, 8:25-32, 1986 .

[CLT96] F. Christensen, M. Lind, and J. Tind. On the nucleolus of NTUgames defined by multiple objective linear programs. ZOR - Mathematical Methods of Operations Research, 43(3):337-352, 1996.

[Ehr95] M. Ehrgott. Lexicographic max-ordering - a solution concept for multicriteria combinatorial optimization. In D. Schweigert, editor, Methods of Multicriteria Decision Theory, Proceedings of the 5th Workshop of the DGOR-Working Group Multicriteria Optimization and Decision Theory, pages 55-66, 1995.

[Ehr96] M. Ehrgott. A characterization of lexicographic max-ordering solutions. Technical report, University of Kaiserslautern, Department of Mathematics, 1996. Report in Wirtschaftsmathematik No. 12. Accepted for Proceedings of the 6th Workshop of the DGOR-Working Group Multicriteria Optimization and Decision Theory.

[Ehr97] M. Ehrgott. Classification and Methodology in Multiple Criteria Optimization. Shaker Verlag, Aachen, 1997. Ph.D. Thesis.

[HY81] C.L. Hwang and K. Yoon. Multiple Attribute Decision Making Methods and Applications. Springer-Verlag, Berlin, Heidelberg, New York, 1981.

[MO92] E. Marchi and J.A. Oviedo. Lexicographic optimality in the multiple objective linear programming: The nucleolar solution. European Journal of Operational Research, 57:355-359, 1992.

[Ogr94] W. Ogryczak. Location problems from the multiple criteria perspective: Efficient solutions. Technical Report 94-019, Université Libre de Bruxelles, 1994. 